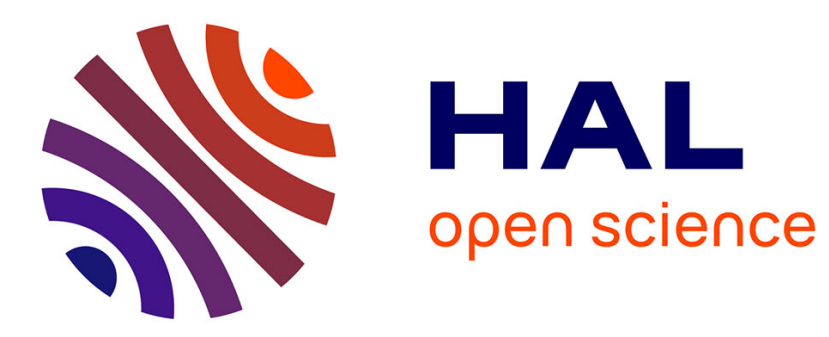

\title{
Evidence of Majorana fermions in the noise characteristic of normal metal-topological superconductor junctions
}

\author{
Thibaut Jonckheere, Jérôme Rech, Laurent Raymond, Alex Zazunov, \\ Reinhold Egger, Thierry Martin
}

\section{To cite this version:}

Thibaut Jonckheere, Jérôme Rech, Laurent Raymond, Alex Zazunov, Reinhold Egger, et al.. Evidence of Majorana fermions in the noise characteristic of normal metal-topological superconductor junctions. Eur.Phys.J.ST, 2020, 229 (4), pp.577-592. 10.1140/epjst/e2019-900119-5 . hal-02497829

\section{HAL Id: hal-02497829 \\ https://hal.science/hal-02497829}

Submitted on 8 Nov 2021

HAL is a multi-disciplinary open access archive for the deposit and dissemination of scientific research documents, whether they are published or not. The documents may come from teaching and research institutions in France or abroad, or from public or private research centers.
L'archive ouverte pluridisciplinaire HAL, est destinée au dépôt et à la diffusion de documents scientifiques de niveau recherche, publiés ou non, émanant des établissements d'enseignement et de recherche français ou étrangers, des laboratoires publics ou privés. 


\title{
Evidence of Majorana fermions in the noise characteristic of normal metal-topological superconductor junctions
}

\author{
T. Jonckheere ${ }^{1, a}$, J. Rech ${ }^{1}$, L. Raymond ${ }^{1}$, A. Zazunov ${ }^{2}$, R. Egger ${ }^{2}$, and T. Martin ${ }^{1}$ \\ 1 Aix Marseille Univ, Université de Toulon, CNRS, CPT, Marseille, France \\ 2 Institut für Theoretische Physik, Heinrich Heine Universität, D-40225 Düsseldorf, Ger- \\ many
}

\begin{abstract}
A finite topological superconductor nanowire bears a Majorana fermion at its ends, leading to unique transport properties when connected to normal metal leads. We consider in this review two theoretical proposals based on noise measurements in normal metal - topological superconductor junctions. The first one considers a Hanbury Brown and Twiss setup where a topological superconductor is connected to two normal metal leads. The second proposal deals with the finite frequency noise of a single normal metal - topological superconductor junction. Both are computed using a unified framework of non equilibrium Keldysh Green's functions using a Hamiltonian approach. Calculations are performed non-perturbatively in the tunnel hopping parameter and address both subgap and above gap regimes. Concerning the Hanbury Brown and Twiss setup, we find in the subgap case that when the two normal metal leads are biased with equal voltage, the noise crossed correlations are negative, as in the case of a three terminal normal metal junction. On the other hand when subgap voltages are opposite, the noise crossed correlations are positive. Predictions when the two Majoranas at the end of the topological superconductors hybridize, and when the chemical potential of the topological superconductor drives the system out of the topological phase are discussed. In the second proposal, the finite frequency emission and absorption noises are computed for a single junction. We observe noticeable structures in these quantities, related to simple transport processes involving the Majorana bound state. Both results offer an original tool for the further characterization of the presence of Majorana bound states in condensed matter systems.
\end{abstract}

\section{Introduction}

The search for the manifestation of Majorana fermions (MF) [1] in condensed matter settings constitutes an on-going and exciting race, both on the theoretical and on the experimental side. In the Kitaev model [2] of a one-dimensional topological superconductor, Majorana fermions emerge at the boundary of a finite chain when specific

\footnotetext{
${ }^{a}$ e-mail: thibaut.jonckheere@cpt.univ-mrs.fr
} 
parameters (nearest neighbor hopping, p-wave pairing amplitude, chemical potential) are precisely tuned. The ground state of the system then becomes degenerate, as the two Majorana bound states (MBS) located on opposite ends of the chain are zero-energy states, corresponding to one delocalized fermion which can be either occupied or empty. This opens the possibility to realize a decoherence-free quantum bit with applications to quantum information schemes. A realistic experimental equivalent of the Kitaev model can be built by inducing s-wave superconductivity in a semiconducting nanowire subject to both Rashba spin-orbit coupling and a Zeeman magnetic field [3-8]. Other proposals where a chain of magnetic atoms are deposited on a superconducting substrate have also been studied $[9,10]$. On the theoretical side, many proposals have been on the market in the last decade [11-16] but despite the significant experimental progress, a smoking gun $[17,18]$ of these Majorana bound states is still lacking today. Most experimental evidence has so far focused on the detection of a zero bias anomaly in the differential conductance, which can occur for multiple reasons other than Majoranas, but a recent claim seems to measure precisely the quantized nature of this low voltage signature [19]. It is nevertheless obvious that more quantum transport tests are needed to put Majorana fermions on a firmer ground, before they can be envisioned to be used for instance in quantum computing schemes. The purpose of the present review is to introduce two new tests based on the measurement of noise (the Fourier transform of the current-current correlation in time) of a normal metal - topological superconductor junction at finite bias.

Since the birth of quantum mesoscopic physics, analogies of electronic transport with quantum optics were pointed out. Laterally confined electrons propagate quasi ballistically via transverse channels as in an optical wave guide (leading to the quantification of the conductance in a quantum point contact), electron interferometers (Fabry-Perot, Mach-Zehnder) have been realized, but also more fundamentally one can imagine reproducing with electrons some celebrated scenarios initiated at the birth of quantum optics such as the Hanbury Brown and Twiss (HBT) experiment [20, 21]. Contrary to bosons, electrons have fermionic statistics, and as charged particles they are likely to interact with the surrounding electronic environment, which promises a variety of rich phenomena. In the original HBT experiment, thermal, monochromatic photons from a mercury arc lamp impinging on a half-silvered mirror led to positive light intensity correlations because the thermal photon source would send on average several photons at a time due to the bunching nature of bosons, resulting in positive intensity correlations. The electronic analog of the HBT experiment with photons consists in a "Y" or "T" shaped structure with a Fermi sea injecting electrons from one arm, with current correlations measured between the two output ports, both with lower chemical potentials. The noise crossed correlations for this device were found to be negative $[22,23]$ : electrons from the Fermi sea input can only end up in one or the other lead. This led to conclusive experimental checks at Basel and at Stanford $[24,25]$. Subsequently, with the advent of noise considerations in mesoscopic superconductivity [26-29], the theoretical community became aware that HBT noise crossed correlations could in principle be achieved for a device consisting of a BCS superconductor as an injector, connected to two normal metal outputs: the Cooper pair beam splitter [27,29-32]. For subgap regimes, Cooper pairs may end up in one lead or the other, by a reverse Andreev reflection process where a hole incident from the normal metal side is reflected as an electron. Alternatively, it can be split with its two constituent electrons propagating separately at the two outputs, a process called crossed Andreev reflection (CAR): a hole incident from one normal metal lead is reflected as an electron into the other normal metal lead. If CAR dominates, positive noise noise crossed correlations were predicted and eventually measured [7]. In particular, in specific devices were quantum dots separate the different leads, it 


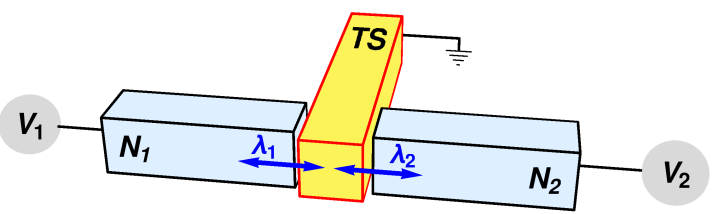

Fig. 1. Schematic view of the setup: a grounded TS nanowire is tunnel coupled (with hopping amplitudes $\left.\lambda_{1}, \lambda_{2}\right)$ to two normal-conducting $\left(\mathrm{N}_{1}, \mathrm{~N}_{2}\right)$ leads which are biased at voltages $V_{1}$ and $V_{2}$, respectively.

was found that the presence of Coulomb interactions in the dots would optimize the positive crossed correlation signal [33].

It is therefore natural to transfer these concepts from quantum optics to hybrid devices containing topological superconductors (hosting MF at their extremities) as a source of electrons. What will be the difference between the BCS case and p-wave pairing? This constitutes the first part of this review and the device that we consider is depicted in Fig. 1. In the context of a scattering approach, it was found [34-36] that a topological superconductor connected to two normal leads at the same subgap voltage would bear negative crossed correlation, in opposition to the BCS superconductor case, and with full correspondence to the all normal lead device of Ref. [22]. In the present work we consider a microscopic model for the normal metal - topological superconductor - normal metal (NTSN) junction with a Hamiltonian formalism. This has two benefits: we are able to treat both subgap and above gap regimes, but more interestingly we also compute the noise crossed correlations for opposite voltages and find them to be positive. The combination of equal and opposite voltages constitutes a novel signature of how the single Majorana fermion operates as a splitter of p-wave Cooper pairs.

We consider here only DC voltages applied to the device, i.e. a continuous stream of electrons emanating from the TS and distributed to the output leads, as opposed to single electron wave packets which are nowadays used in the field of electron quantum optics [37]. Our choice is in close analogy with single photon sources [38] which bear negative correlations at the output of a half silvered mirror because of partitioning.

In the second part of this review, we shall focus on single normal metal - topological superconductor (NTS) junction, but this time we will seek signatures of Majorana fermions via finite frequency noise measurements. Finite frequency noise has been studied in the context of quantum mesoscopic physics and nano-physics, both theoretically and experimentally. On the theoretical side, it has been argued [39-41] that since the two current operators entering the noise correlator are evaluated at different times, one can introduce two quantities - the emission and the absorption noise, because photons from the nanodevice under study may be emitted to the detector, and the latter, which can in practice have a higher temperature than the nanodevice, may emit photons which will be absorbed by the nanodevice. A finite frequency noise detector should be described quantum mechanically, at the same level as the nanodevice under study. The measured signal by the quantum detector, which is a real number, should thus be a combination of such emission and absorption quantities. This has been experimentally verified in pioneering finite frequency measurement experiments performed in Deflt [42], in Orsay [43-45] and in Saclay [46] in different contexts. In a previous work, the finite frequency noise of a normal metal - BCS superconductor junction $[28,47]$ was computed, and cusps or singularities were found at specific frequencies corresponding to different transport processes. However, one can expect different results in the case of the NTS junction, as the density of states 
(DOS) of the topological superconductor differs substantially from its conventional BCS counterpart. The BCS density of states is zero inside the gap, and bears inverse square root divergences at the gap edges. On the opposite, the DOS of a TS wire has a square root behavior outside the gap and, more importantly, a so-called Majorana peak at zero energy. It can therefore be expected that the finite frequency noise of an NTS junction may bear peculiar features associated with the Majorana bound state embedded in the junction.

The review is organized as follows. In Sec. 2, the model and formalism are introduced; Sec. 3 is devoted to the HBT setup, while the finite frequency noise of the NTS junction is presented in Sec. 4.

\section{Model and Formalism}

\subsection{Hamiltonian for the TS wire}

For the sake of generality, we consider a multi-terminal device in a T-shaped geometry, as illustrated in Fig. 1, where one end of a topological superconductor (TS) nanowire is contacted by several (two at most) normal-conducting (N) leads. Voltages $V_{1,2}$ are applied across the N-TS contacts, while the TS wire is grounded. The full Hamiltonian is given by

$$
H=H_{T S}+H_{N}+H_{t},
$$

where the first two terms describe the TS and two N-leads, respectively, and $H_{t}$ is a tunneling Hamiltonian connecting all leads to each other. The TS Hamiltonian corresponds to the continuum version of a Kitaev chain $[2,11]$ in the wide-band limit, and reads for $x>0$ :

$$
H_{T S}=\int_{0}^{\infty} d x \Psi_{T S}^{\dagger}(x)\left(-i v_{F} \partial_{x} \sigma_{z}+\Delta \sigma_{y}\right) \Psi_{T S}(x),
$$

where $\Delta$ is a proximity-induced pairing gap, assumed to be real, the Nambu spinor $\Psi_{T S}(x)=\left(c_{r}, c_{l}^{\dagger}\right)^{T}$ combines right- and left-moving fermions, with annihilation field operators $c_{r}(x)$ and $c_{l}(x)$, respectively, $v_{F}$ is the Fermi velocity and $\sigma_{x, y, z}$ are the Pauli matrices in Nambu space. We use units with $k_{B}=v_{F}=\hbar=1$.

The transport problem is described in terms of boundary Keldysh Green functions (GFs) [48] describing the leads which are coupled together by tunneling processes. The exact boundary GFs can be obtained by solving the Dyson equation to all orders in the tunnel couplings.

\subsection{Green's functions}

The bare boundary Keldysh GF at $x=0$ for the TS wire is defined as follows:

$$
\check{g}_{T S}\left(t-t^{\prime}\right)=-i\left\langle\mathcal{T}_{C} \Psi(t) \Psi^{\dagger}\left(t^{\prime}\right)\right\rangle
$$

where the Nambu spinor $\Psi=\left(c, c^{\dagger}\right)^{T}$ contains the boundary fermion operator $c=$ $\left[c_{l}+c_{r}\right](x=0)$, and $\mathcal{T}_{C}$ denotes Keldysh time ordering. The Fourier transforms of the retarded and advanced GFs for the uncoupled TS wire in the topologically nontrivial phase [48] are

$$
g_{T S}^{R / A}(\omega)=\frac{\sqrt{\Delta^{2}-\left(\omega \pm i 0^{+}\right)^{2}} \sigma_{0}+\Delta \sigma_{x}}{\omega \pm i 0^{+}},
$$


where $R / A$ corresponds to $+/-$ and $\sigma_{0}$ is the unity matrix in Nambu space. This simple expression for the retarded/advanced boundary GF of a TS wire captures the zero-energy Majorana bound state (resulting in a zero energy peak in the density of states, as depicted in the lower left inset of Fig. 4) as well as continuum quasiparticles, which allows for studying both subgap and above-gap transport on equal footing. The Keldysh component $g_{T S}^{K}(\omega)$ is expressed via the retarded and advanced components as:

$$
g_{T S}^{K}(\omega)=\left[1-2 n_{F}(\omega)\right]\left[g_{T S}^{R}(\omega)-g_{T S}^{A}(\omega)\right],
$$

where $n_{F}(\omega)=\left(e^{\omega / T}+1\right)^{-1}$ is the Fermi function with temperature $T$. Throughout the paper we use the chemical potential $\mu_{T S} \equiv 0$ of the (grounded) TS wire as a reference energy level. In the appendix of Ref. [49], one can find a derivation of the boundary Green function for a Kitaev chain with the finite bandwidth and arbitrary values for the band filling, while the wide-band expression of Eq. (4) exhibiting particle-hole symmetry corresponds to half filling.

In the same manner we construct the Keldysh GFs for the normal leads by setting $\Delta=0: g_{N}^{R / A}(\omega)=\mp i \sigma_{0}$, The Keldysh component $g_{N}^{K}(\omega)$ is also specified, and we choose to include the DC bias in the Fermi distribution of electrons and holes: $g_{N}^{K}(\omega)=-2 i\left[1-2 n_{F}\left(\omega-\mu_{N} \sigma_{z}\right)\right] \sigma_{0}$ (the chemical potential $\mu_{N}$ is shifted with respect to $\mu_{T S}=0$ by the DC voltage across the NTS junction).

\subsection{Tunnel Hamiltonian}

In what follows, we consider for simplicity equal couplings $\lambda$ from the TS wire to the normal leads, and no direct coupling between the normal leads. This is by no means a restriction of our formalism as the general case is treated in Ref. [49]. The tunneling Hamiltonian is expressed in terms of the boundary fermions $c_{j}$ (with $j=0$ for the TS wire and $j=1,2$ ) representing the leads [48]:

$$
H_{t}=\frac{1}{2} \sum_{j, j^{\prime}} \Psi_{j}^{\dagger} W_{j j^{\prime}} \Psi_{j^{\prime}}
$$

with $\Psi_{j}=\left(c_{j}, c_{j}^{\dagger}\right)^{T}$ the boundary Nambu spinor and $W=W^{\dagger}$ is the tunneling matrix in lead and Nambu space whose elements are given by $W_{j j^{\prime}}=\lambda_{j j^{\prime}} \sigma_{z}$. For the present setup, this is further simplified as

$$
W=\left(\begin{array}{ccc}
0 & \lambda \sigma_{z} & \lambda \sigma_{z} \\
\lambda \sigma_{z} & 0 & 0 \\
\lambda \sigma_{z} & 0 & 0
\end{array}\right)
$$

The full Keldysh GF $\check{G}$ of the system follows from the Dyson equation

$$
\check{G}=\left(\check{g}^{-1}-\check{W}\right)^{-1},
$$

with the Keldysh matrix $\check{W}=\operatorname{diag}(W,-W)$, and the "uncoupled" Keldysh GF $\check{g}$, diagonal in lead space.

From the tunneling Hamiltonian, Eq. (6), the current operator flowing through lead $j$ reads:

$$
\hat{I}_{j}(t)=i \frac{e}{\hbar} \sum_{j^{\prime} \neq j} \Psi_{j}^{\dagger}(t) \sigma_{z} W_{j j^{\prime}} \Psi_{j^{\prime}}(t)
$$




\section{Hanbury Brown and Twiss correlations}

\subsection{Analytical and numerical results}

The HBT correlations are readily obtained through this formalism by computing the zero-frequency cross-correlations of the above-defined current operator. The current correlations at zero frequency read:

$$
S_{j j^{\prime}}=\int_{-\infty}^{\infty} d \tau\left\langle\delta \hat{I}_{j}(\tau) \delta \hat{I}_{j^{\prime}}(0)\right\rangle
$$

with $\delta \hat{I}_{j}(t)=\hat{I}_{j}(t)-I_{j}$, and $I_{j}=\left\langle\hat{I}_{j}(t)\right\rangle$ is the average current. In terms of the full $\mathrm{GF}$, these current correlations are given by:

$S_{j j^{\prime}}=e^{2} \int_{-\infty}^{\infty} \frac{d \omega}{2 \pi} \sum_{j_{1} \neq j} \sum_{j_{2} \neq j^{\prime}} \operatorname{Tr}_{N}\left\{\lambda_{j j_{1}}\left[G_{j_{1} j_{2}}^{-+}(\omega) \lambda_{j_{2} j^{\prime}} G_{j^{\prime} j}^{+-}(\omega)-G_{j_{1} j^{\prime}}^{-+}(\omega) \lambda_{j^{\prime} j_{2}} G_{j_{2} j}^{+-}(\omega)\right]\right\}$,

where $G^{-s s}=(1 / 2)\left[G^{K}+s\left(G^{R}-G^{A}\right)\right]$ with $s= \pm$.

One can then derive an explicit form for the average current $I_{j}$ the local and the non-local differential conductance (not shown, see Ref. [49]), and the current correlations $S_{j j^{\prime}}$. The expressions we give below depend on the coupling $\lambda$ between the TS and the normal electrodes 1 and 2, on the voltages of the two normal electrodes, and on the temperature through the Fermi function $n_{F}(x)$. A natural quantity appearing in these formulas is $\Lambda=\sqrt{2 \lambda^{2}}$, which is related to the total transmission probability $\tau$ between the TS and the normal leads: $\tau=4 \Lambda^{2} /\left(1+\Lambda^{2}\right)^{2}$. For all the results presented in this work, we focus on the case $0<A<1$, which covers all possible values of the transmission $\tau \in[0,1]$.

General expressions for the noise crossed correlations can be obtained for arbitrary voltages [49]. Here for simplicity we provide expressions for the case of equal and opposite voltages. Introducing the broadening $\Gamma=2 \Delta \Lambda^{2} /\left(1-\Lambda^{4}\right)$, we have, for equal voltages:

$$
S_{12}(V, V)=-\frac{2 e^{2}}{h} \frac{\Gamma^{2}}{4} \frac{|e V|}{(e V)^{2}+\Gamma^{2}},
$$

which coincides with existing results.[34,35] Conversely, for opposite voltages, we have:

$$
S_{12}(-V, V)=\frac{2 e^{2}}{h} \frac{\Gamma^{2}}{4}\left[\frac{|e V|}{(e V)^{2}+\Gamma^{2}}+\frac{2 \Gamma^{2}+(e V)^{2}}{(e V)^{2}+\Gamma^{2}} \frac{|e V|}{\Delta^{2}}-\frac{2 \Gamma}{\Delta^{2}} \tan ^{-1}\left(\frac{|e V|}{\Gamma}\right)\right] .
$$

Fig. 2 shows the HBT cross-correlation noise $S_{12}$ for equal and opposite voltages, computed for three different values of the transmission probability $\tau$. One can see that the cross-correlations in these two cases are simply opposite as long as $\mathrm{eV} \ll \Gamma$, with negative (positive) values of $S_{12}$ for the equal (opposite) voltage case. For $|e V|$ larger than the gap $\Delta, S_{12}$ is always a decreasing function of $|V|$, which, for the opposite voltage case, eventually becomes negative for $|e V| \gg \Delta$ (not shown), as the TS behaves essentially as a normal electrode at such high voltages.

In the limit of small voltages $|V| \ll \Gamma, S_{12}$ becomes

$$
S_{12}( \pm V, V) \simeq \mp \frac{1}{2} \frac{e^{2}}{h}|e V|
$$

Importantly, this means that the HBT cross-correlations are positive when the two voltages have opposite signs. 


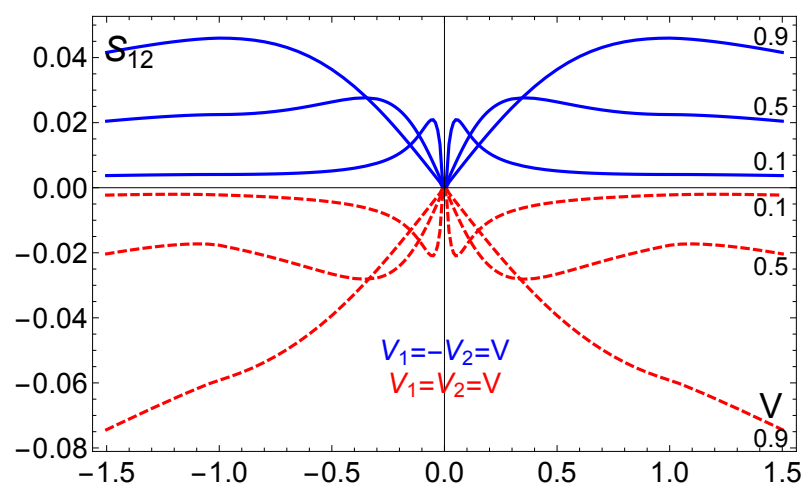

Fig. 2. HBT cross-correlations $S_{12}$ (in units of $e^{2} / h$ ) vs $V$ in the case of equal (red dashed curve) and opposite (blue full curve) voltages for equal couplings and several transparencies $\tau$ (indicated in black on the left), at zero temperature.

\subsection{Discussion of auto- and crossed- correlations}

In order to get an intuitive picture of the noise crossed correlations $S_{12}$, it is useful to discuss also the noise autocorrelation contributions, $S_{00}, S_{11}$ and $S_{22}$. They obey a conservation law [22] arising from current conservation:

$$
S_{00}=S_{11}+S_{22}+2 S_{12}
$$

For simplicity, we consider in what follows the regime of symmetric couplings in the zero-temperature limit.

\subsubsection{Equal voltages}

The analytical expressions for the various noise correlations (in units of $e^{2} / h$ ) are:

$$
\begin{aligned}
& S_{00} \simeq 0 \\
& S_{11} \simeq \frac{|e V|}{2} \\
& S_{12} \simeq-\frac{|e V|}{2}
\end{aligned}
$$

in the low-voltage limit $e V \ll \Gamma$.

From the point of view of the TS, the two normal electrodes are at the same potential and thus act as a single one for the total current $I_{0}$, so that the total conductance has a peak of height $2 e^{2} / h$ (see Ref. [48]). As a consequence, much like in the single N-TS junction, the total current $I_{0}$ is noiseless at low voltage $\mathrm{eV} \ll \Gamma$, which is confirmed by Eq. (16). This total current $I_{0}=2\left(e^{2} / h\right) V$ is partitioned here with equal probability between the currents $I_{1}$ and $I_{2}$ (see Fig. 3) :

$$
I_{1}=I_{2}=\frac{e^{2}}{h} V
$$

These two currents are thus equivalent to the transmitted and backscattered current from a quantum point contact with incoming current $I_{0}$ and transmission $T=1 / 2$, reflection $R=1-T=1 / 2$. 


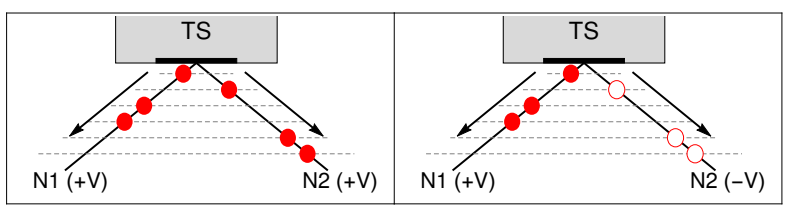

Fig. 3. Schematic picture of transport in the NTSN junction at low voltage. Electrons (holes) are shown as full (empty) circles. Left panel: equal voltages, with perfect anti-correlations of the two electron streams. Right panel: opposite voltages so that electrons (holes) are emitted into $\mathrm{N}_{1}\left(\mathrm{~N}_{2}\right)$. Arrows indicate directions of quasiparticle motion.

This implies that the autocorrelations $S_{11}$ and $S_{22}$ correspond to the noise associated with currents resulting from random partitioning [50], leading to (restoring units):

$$
S_{j j} \equiv e I_{j}(1-T)=\frac{e^{2}}{h} \frac{|e V|}{2} \quad(j=1,2)
$$

which coincides with Eq. (17) to lowest order in $V$.

The HBT noise $S_{12}$ corresponds to the correlation between the two partitioned currents $I_{1}$ and $I_{2}$. Due to the fermionic nature of the electrons, these two currents are totally anti-correlated (see Fig. 3), yielding a negative correlation noise [22, 23, 51]. Following Eq. (15), and using that $I_{0}$ is noiseless, one sees that the HBT correlations and the autocorrelations are simply related as $S_{12}=-S_{11}$, which agrees with Eq. (18).

\subsubsection{Opposite voltages}

For opposite voltages, the auto- and cross-correlations in the limit $\mathrm{eV} \ll \Gamma$ (in units of $\left.e^{2} / h\right)$ take the form:

$$
\begin{aligned}
& S_{00} \simeq 2|e V| \\
& S_{11} \simeq \frac{|e V|}{2} \\
& S_{12} \simeq \frac{|e V|}{2}
\end{aligned}
$$

This opposite voltage case can nevertheless still be understood on the same footing as the equal voltage case by considering that the coupling to the Majorana bound state is perfectly electron-hole symmetric. Normal lead 2 (at voltage $-V$ ) can be viewed as a reservoir of holes (biased at voltage $V$ ) coupled to the Majorana bound state: electrons are now replaced by holes for the current $I_{2}$. The total current from the TS is a stream of particles (electrons or holes), which is still noiseless, with one particle (electron or hole) emitted during each time interval $\hbar / \mathrm{eV}$. The currents $I_{1}$ and $I_{2}$ still result from the random partitioning of such a noiseless stream of particles, with electrons for $I_{1}$ and holes for $I_{2}$, so that

$$
I_{1}=-I_{2}=\frac{e^{2}}{h} V
$$

As a consequence the autocorrelation noises $S_{11}=S_{22}=\left(e^{3} / h\right)|V| / 2$ are identical to their counterparts in the equal voltage case (to lowest order in $e V / \Gamma$ ). As in the equal voltage case, the two currents $I_{1}$ and $I_{2}$ are totally anti-correlated, which leads to the same expression for the HBT correlation noise $S_{12}$, only with the opposite sign, as the carriers in the two leads now bear opposite charges (see Fig. 3). 

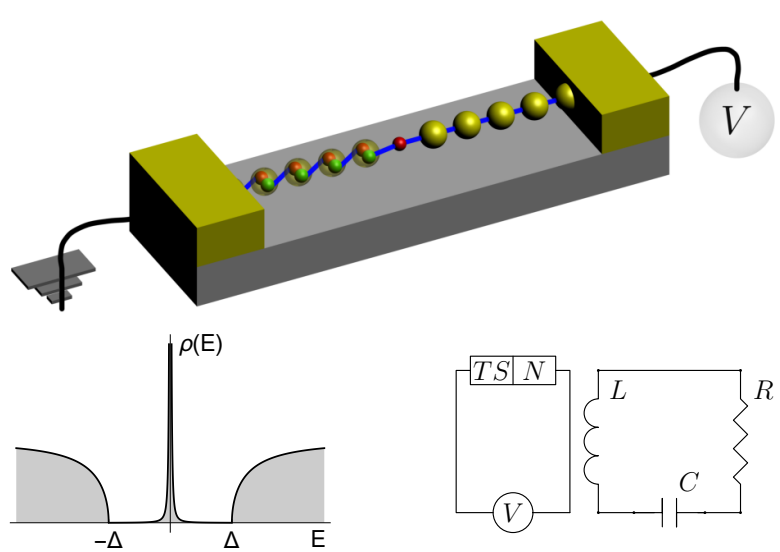

Fig. 4. Top: schematic view of a junction between a normal metal (yellow dots) and a topological superconductor (red-green dots, with a red Majorana bound state at its end); the normal metal is biased at voltage $V$. Bottom left: topological superconductor density of states, with a central peak at zero energy due to the Majorana bound state, and square root behavior outside the gap. Bottom right: the principle of the noise detection, using the inductive coupling to a RLC circuit [39].

Finally the total noise $S_{00}$ (which accounts for the charge of the carriers) corresponds to the current noise of a noiseless stream of particles (one particle -electron or hole- transmitted at each time interval $\hbar / \mathrm{eV}$ ) but with particles which can be either electron or holes. According to Eq. (15) this creates a total charge noise $S_{00}=2\left(e^{3} / h\right)|V|$, which coincides with Eq. (21).

The equal and opposite voltage regimes thus have cross-correlations with opposite signs, a peculiarity of the Majorana bound state, which does not distinguish electrons from holes.

\subsection{Finite length and doping effects}

Using the same Green functions formalism, and adapting the boundary Green function used for the TS nanowire, we were also able (not shown) to consider more general situations. We considered a TS nanowire of finite length and also studied the effect of varying the chemical potential [49].

For the finite length case, our results show the existence of a crossover when the length $L$ becomes smaller than $\hbar v_{F} / \Delta$ (the typical decay length associated with the Majorana bound state), with positive correlations becoming negative for small lengths. This behavior is due to the hybridization of the two Majoranas at the ends of the TS nanowire, which then behave as a regular fermion. This confirms that the unusual sign observed for the current correlations for a long (or semi-infinite) TS nanowire is specific to the presence of a Majorana bound state.

For the impact of the TS chemical potential, we considered the case where the TS nanowire is represented by a Kitaev chain with a finite bandwidth, and variable chemical potential $\mu$ (the corresponding boundary Green function is derived in Ref. [49]). By varying $\mu$, one can explore the transition from the topological to the trivial phase. Our results show that above the transition, the cross-correlations become negative at all voltages, and the specific features due to the Majorana bound state (peak at low voltage) disappear. 


\section{Finite frequency noise of an NTS junction}

\subsection{Emission, absorption, and measurable noise}

We now consider the finite frequency noise in a NTS junction. While general formulas valid at arbitrary transparency can be obtained [52], the most relevant regime is the case of low transparency, where the junctions properties are more visible in the emission/absorption noise. For the analysis of the finite frequency noise, one has to consider separately the two real time noise correlators corresponding to emission and absorption, whose Fourier transform read:

$$
\begin{aligned}
& S^{+}(\Omega)=\int_{-\infty}^{+\infty} d t\langle\delta I(0) \delta I(t)\rangle \mathrm{e}^{i \Omega t}, \\
& S^{-}(\Omega)=\int_{-\infty}^{+\infty} d t\langle\delta I(t) \delta I(0)\rangle \mathrm{e}^{i \Omega t}=S^{+}(-\Omega) .
\end{aligned}
$$

$S^{+}(\Omega)$ is conveniently rewritten using the Fourier-transformed dressed Green's functions as:

$$
S^{+}(\Omega)=\lambda^{2} e^{2} \int_{-\infty}^{+\infty} \frac{d \omega}{2 \pi} \operatorname{Tr}_{N}\left[G_{00}^{-+}(\omega) G_{11}^{+-}(\omega+\Omega)-G_{01}^{-+}(\omega) G_{01}^{+-}(\omega+\Omega)\right]
$$

In order to describe the whole range of physical parameters, we can safely focus on positive frequencies for both noise spectra.

In order to connect with potential experimental works looking to investigate the finite-frequency noise of the NTS junction, we can combine the emission and absorption noise spectra to obtain the measurable noise associated with a generic quantum detector consisting of a resonant LC circuit, inductively coupled to the NTS junction (see Fig. 4 lower right inset). This measured noise is the result of repeated measurements of the charge at the capacitor plates. For the present setup, we use an expression formulated in Refs. $[39,53]$ to predict the result of such a measurement:

$$
S_{\text {meas }}(\Omega)=K\left\{S^{+}(\Omega)+N(\Omega)\left[S^{+}(\Omega)-S^{-}(\Omega)\right]\right\},
$$

where $N(\Omega)=\left[\exp \left(\hbar \Omega / k_{B} T_{L C}\right)-1\right]^{-1}$ is the Bose-Einstein distribution associated with the oscillator modes, and $K$ is the effective inductive coupling constant of the quantum wire with the resonator.

\subsection{Qualitative picture}

An intuitive picture of the emission and absorption noise processes can be gained by looking at the occupied states of both the $\mathrm{N}$ and the TS side of the junction and by identifying (to lowest order in the tunneling amplitude) which electron transport contribute to both noises. These are depicted in Fig. 5. The two processes of the top row ( $\mathrm{a}$ and $\mathrm{b}$ ) describe emission noise, while the four other processes describe absorption noise. Here we choose $\mathrm{eV}>0$ without loss of generality.

When the bias voltage satisfies $e V<\Delta$, there is only one process contributing to the emission noise: panel (a) of Fig. 5. Electrons from $\mathrm{N}$ with energies in the interval $[0, e V]$ may be transferred on the TS side to the MF bound state by emitting a photon of energy $\Omega=e V$. As the width of the Majorana peak is negligible in the small transparency regime, and the DOS of $\mathrm{N}$ is approximatively constant, this emission process in independent of the frequency. 

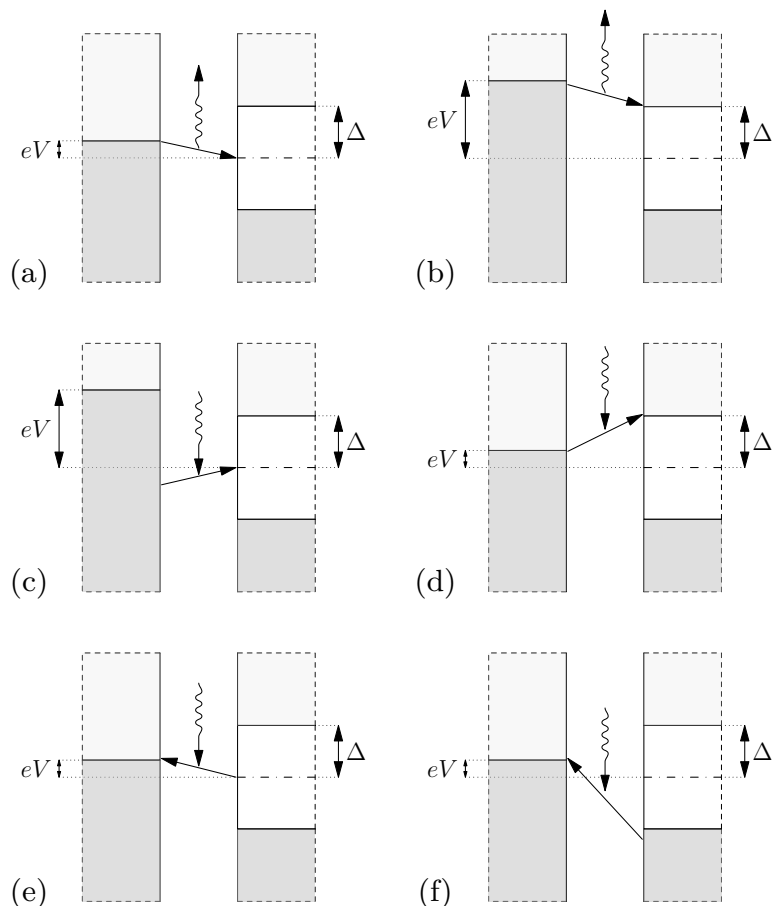

Fig. 5. Energetic diagram of a NTS junction, with occupied electronic states (medium grey) and empty states (light grey). (a)-(b) emission processes, (b) is not available for a bias $\mathrm{eV}$ below the gap $\Delta$, (c)-(d) absorption processes involving a transition from the metal to the superconductor, (e)-(f) absorption processes with transitions to the metal. Straight lines correspond to the transfer of electrons while wiggly lines describe the absorption or emission of photons.

For voltages larger than the gap, another emission process is available: electrons from $\mathrm{N}$ can be transferred to the empty states above the TS gap, and emit a photon in the range $[0, \mathrm{eV}-\Delta]$ (see Fig. $5 \mathrm{~b}$ ). The frequency dependence of this process reflects the energy dependence of the TS density of states above the gap.

Absorption processes can also be realized [see panels (c) and (d) of Fig. 5], and show similar frequency dependence as their emission counterparts. In the case of panel (c), an electron from $\mathrm{N}$ absorbs a photon to be transmitted to the Majorana state. Panel (d) is the equivalent of panel (b) for photon absorption: an electron from the normal metal absorbs a photon and is transmitted to an empty state above the TS gap. Note processes (a) and (c) exist for any voltages, but on the opposite the emission process of panel (b) can only occur for $\mathrm{eV}>\Delta$.

Finally, photon absorption can involve electrons which are transmitted to $\mathrm{N}$ from the topological superconductor, for frequencies in the interval $[e V,+\infty]$, as displayed in panels (e) and (f) of Fig. 5. The TS electron is transmitted to a normal metal empty state, either from the Majorana state [panel (e)] or from the occupied states below the gap [panel (f)].

\subsection{Above gap regime}

We are able to treat in full generality both the subgap and above gap regimes for the emission and absorption noise of the NTS junction[52]. We decide here to focus on 

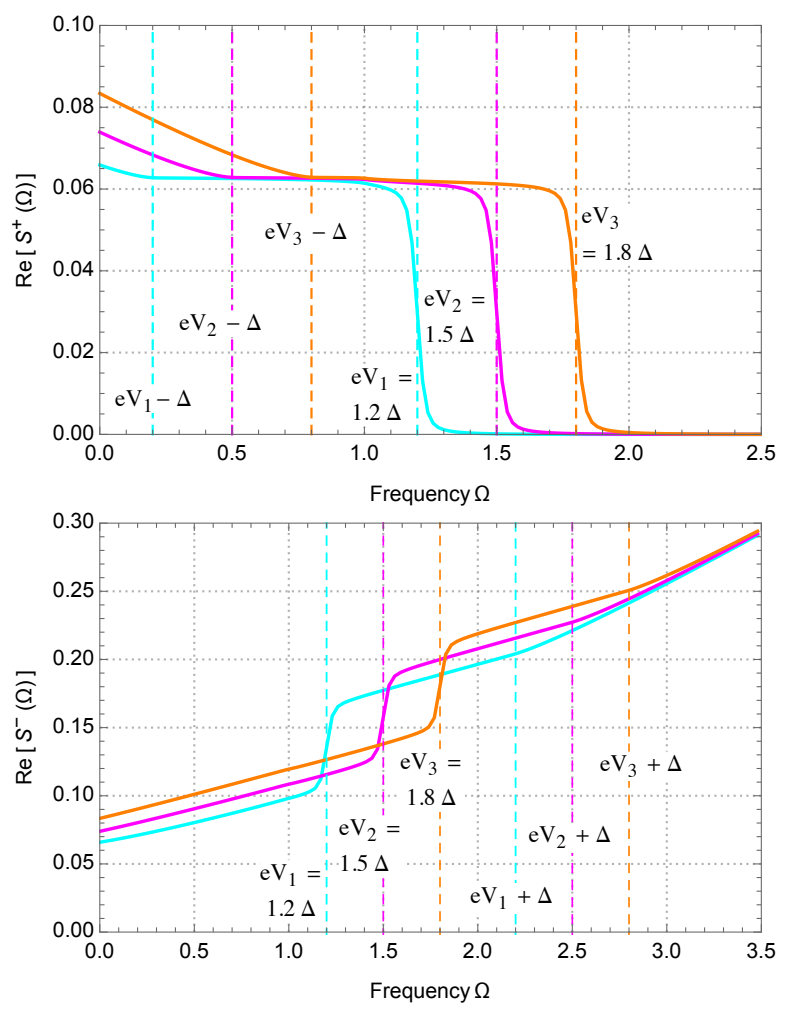

Fig. 6. Emission noise $S^{+}(\Omega)$ (top) and absorption noise $S^{-}(\Omega)$ (bottom) as a function of frequency $\Omega$ (in units of $\Delta$ ) at low transparency $(\tau=0.02)$ computed beyond the gap $|e V|>\Delta$ for three different values of the voltage bias $(e V=1.2 \Delta, 1.5 \Delta$ and 1.8 $\Delta)$, and expressed in units of $e^{2} \Delta / h$.

the above gap transport, which encompasses more transport processes according to the qualitative picture of the preceding section.

We plot the emission noise $S^{+}(\Omega)$ in Fig. 6 (top), for three different voltages. As in the subgap regime (not shown, see Ref. [52]), the emission noise contains a welldefined plateau extending - for the above gap situation - in the interval $[\mathrm{eV}-\Delta, \mathrm{eV}]$, dropping to zero beyond this. This plateau reflects the process shown in Fig. 5a (it is expected to lead to a constant emission noise, restricted to frequencies $\Omega<e V$ because of electron occupancy). This contribution persists at low frequency, but it is supplemented by process (b) (see Fig. 5) leading to an increase of the noise when the frequency is decreased, as the DOS of the TS increases beyond the gap. This constitutes a novel noise feature compared to the subgap regime.

The above gap absorption noise is shown in Fig. 6 (bottom). Again a sharp step occurs for $\Omega=e V$, associated with process (e) of Fig. 5. A smaller structure, visible at $\Omega=\Delta+e V$, is tied to the onset of process (f). Processes (c) and (d) also contribute to the absorption noise, being present at all frequencies and leading respectively to a constant background contribution and to a steady increase of the noise.

The main message is therefore that for voltages both above and below the TS gap, the presence of a Majorana bound state leads to a distinctive plateau in the emission noise as a function of frequency, accompanied by a sharp drop to zero at $\Omega=e V$. We are able to relax the constraint of low transparencies, and find that higher transparencies tend to smooth out the noise structures defined above (see Ref. [52]). 
Tunnel NTS junctions are therefore the best candidates for the observation of the finite frequency noise signatures of the Majorana bound state.

\subsection{Measurable noise}

In order to analyze the measurable noise, we choose the inductive coupling scheme introduced in Ref. [39] which involves an LC circuit in the vicinity of the NTS junction (see Fig. 4). This protocol relies on repeated measurements of the charge on the plates of the capacitor of the LC resonant circuit (the latter bearing its own temperature $T_{L C}$ ). When the detector temperature is such that $T_{L C} \ll \Omega$, the measurable noise closely resembles the emission noise as the Bose-Einstein distribution becomes vanishingly small, so that the measurable noise reduces to:

$$
S_{\text {meas }}\left(\Omega \gg T_{L C}\right) \simeq K S^{+}(\Omega) .
$$

This means in practice that when the temperature of the detector $T_{L C}$ is comparable to the temperature of the NTS circuit (which is here assumed to be chosen well below the gap $\Delta$ ), the shape of the measurable noise is identical to the top panel of Fig. 6 .

As one increases the detector temperature, choosing for instance $T_{L C}=\Delta$, the direct connection with the emission noise is damaged, ultimately leading to the distortion of the noise plateau (see Ref. [52], not shown). Nevertheless, the typical onsets at frequencies $\Omega=\mathrm{eV}-\Delta$ and $\Omega=\mathrm{eV}$ are still easy to identify: the measurable noise shows a sudden change of sign near $\Omega=e V$, attributed to the sharp drop in the emission noise, which is a consequence of the Majorana peak in the TS DOS. The measurable noise associated to a high $T_{L C}=\Delta$ can therefore still be used as a tool to uncover signatures of the MF.

\subsection{Comparison with a non-topological setup containing zero energy Andreev bound states}

Could the same noise characteristics be obtained from a non-topological superconductor/normal metal junction bearing zero-energy Andreev bound states (ABS) ? We attempt to answer this question by considering a normal metal - quantum dot - BCS superconductor (NDS) junction. First, the parameters of this junction should reproduce the zero bias anomaly in the differential conductance of the NTS junction, due to the presence of a zero energy ABS. Second, we should inquire whether the emission and absorption noise characteristics of such a NDS junction are distinct from the NTS case.

In Ref. [54] which dealt with the BCS superconducting beam splitter, we performed noise crossed correlations calculations on such devices using a path integral approach, which allowed to derive analytical expressions for the currents and the noise crossed correlations in terms of the full Green's function (dressed by the tunneling couplings) of the quantum dots. There, we found that in a junction containing a superconducting source of Cooper pairs connected to two normal leads (which are separated by quantum dots for purpose of energy filtering), the energy levels of the dots could be tuned (anti-symmetrically) in order to favor positive noise correlations, a signature of Cooper pair splitting [29]. Here we can simply adapt this path integral formalism in order to describe a single NDS junction in a non-topological regime. This requires minimal adaptation [52] as noise auto-correlations were not computed there [54].

We need to specify parameters of the NDS system for which the conductance yields a conductance peak at zero energy, similar to the one obtained for a NTS 
junction due to the presence of the Majorana bound state. This is achieved when two conditions are satisfied: setting the quantum dot energy to the superconducting chemical potential, and using symmetrical couplings for the dot between the normal metal and the BCS superconductor. This symmetric junction leads to a conductance peak which is identical to the one of a NTS junction: if tunnel couplings differ, the zero bias conductance shows a split peak away from zero energy.

We now focus on the emission noise characteristics of the NDS device. Indeed, for the NDS system [52] there is also a well identified plateau in the noise extending to $\Omega=e V$, which is a consequence of the discrete quantum dot level. However, at low frequency, in the vicinity of the dot energy broadening, there is a dip in the emission noise, with a value at zero frequency which is exactly half that of the plateau: when probed on a long timescale (by zero frequency noise), the current fluctuations are reduced by a factor $1 / 2$ due to the double-barrier structure with symmetric couplings (which are essential for the zero bias anomaly in the conductance). This factor $1 / 2$ for the contrast of the observed dip corresponds to the well-known Fano factor reduction in double-barrier symmetric junctions [55].

This dip in the emission noise is completely absent in the case of a NTS junction, because the Majorana bound state is an intrinsic part of the superconductor, and is not separated by a barrier. The low-frequency behavior of the emission noise is therefore able to discriminate between the NTS and the NDS system. We therefore claim that the frequency noise characteristics obtained in the NTS cannot be attributed to the presence of a zero energy ABS in a symmetric NDS junction.

\section{Conclusion}

To summarize, two distinctive noise diagnostics have been presented for NTS junctions in this review. First, an HBT noise crossed correlations which exhibits, in the subgap voltage regime, negative noise crossed correlations for equal voltages on the $\mathrm{N}$ leads (in correspondence with a setup containing three $\mathrm{N}$ leads), and positive noise crossed correlations when the voltage of the $\mathrm{N}$ leads are opposite (in correspondence with the signal of a BCS Cooper pair beam splitter with equal voltages on the $\mathrm{N}$ leads). Of course, the sign of the noise crossed correlations has nothing in common with normal metal forks or the BCS Cooper pair splitter, as the MF in the NTS junction is specific to topological matter and treats electrons and holes on the same footing. Extensions to above gap voltages, to a finite size TS nanowire where the two MF hybridize (because the coherence length becomes comparable to the size of the TS), and for a TS driven in the non-topological phase, have been discussed.

Second, we have derived the emission and absorption finite frequency noise of a single NTS junction, and we have shown that both contain distinctive features (onsets and the distinctive noise plateau) which cannot be attributed to a non-topological superconductor junction with a $\mathrm{N}$ lead bearing a zero energy ABS. Contrary to a diagnostic based on the low voltage properties (the zero bias peak and the quantized differential conductance), both proposals rely on finite voltage scenarios (voltages which are comparable to the superconducting gap). We believe that these two results offer further evidence of how MF could operate in electronic quantum transport experiments.

Acknowledgements : The second part of this work was part of the Masters thesis of Didier Bathellier at Aix Marseille University. The project leading to this publication has received funding from Excellence Initiative of Aix-Marseille University A*MIDEX, a French "Investissements d'Avenir" program. 


\section{References}

1. E. Majorana, Nuovo Cim 14, 171 (1937)

2. A.Y. Kitaev, Physics-Uspekhi 44, 131 (2001)

3. R.M. Lutchyn, J.D. Sau, S. Das Sarma, Phys. Rev. Lett. 105, 077001 (2010)

4. Y. Oreg, G. Refael, F. von Oppen, Phys. Rev. Lett. 105, 177002 (2010)

5. J. Alicea, Y. Oreg, G. Refael, F. von Oppen, M.P.A. Fisher, Nature Physics 7, 412 (2011)

6. V. Mourik, K. Zuo, S.M. Frolov, S.R. Plissard, E.P.A.M. Bakkers, L.P. Kouwenhoven, Science 336, 1003 (2012)

7. A. Das, Y. Ronen, Y. Most, Y. Oreg, M. Heiblum, H. Shtrikman, Nature Physics 8, 887 (2012)

8. R.M. Lutchyn, E.P.A.M. Bakkers, L.P. Kouwenhoven, P. Krogstrup, C.M. Marcus, Y. Oreg, Nature Reviews Materials 3, 52 (2018)

9. S. Nadj-Perge, I.K. Drozdov, J. Li, H. Chen, S. Jeon, J. Seo, A.H. MacDonald, B.A. Bernevig, A. Yazdani, Science 346, 602 (2014)

10. H. Kim, A. Palacio-Morales, T. Posske, L. Rózsa, K. Palotás, L. Szunyogh, M. Thorwart, R. Wiesendanger, Science Advances 4 (2018), https://advances.sciencemag.org/content/4/5/eaar5251.full.pdf

11. J. Alicea, Reports on Progress in Physics 75, 076501 (2012)

12. M. Leijnse, K. Flensberg, Semiconductor Science and Technology 27, 124003 (2012)

13. C. Beenakker, Annual Review of Condensed Matter Physics 4, 113 (2013)

14. T.D. Stanescu, S. Tewari, Journal of Physics: Condensed Matter 25, 233201 (2013)

15. R. Aguado, Riv. Nuovo Cimento 40, 523 (2017)

16. S.R. Elliott, M. Franz, Rev. Mod. Phys. 87, 137 (2015)

17. S. Kashiwaya, Y. Tanaka, Reports on Progress in Physics 63, 1641 (2000)

18. P. Burset, B. Lu, S. Tamura, Y. Tanaka, Phys. Rev. B 95, 224502 (2017)

19. H. Zhang, C.X. Liu, S. Gazibegovic, D. Xu, J.A. Logan, G. Wang, N. van Loo, J.D.S. Bommer, M.W.A. de Moor, D. Car et al., Nature 556, 74 (2018)

20. R.H. Brown, R.Q. Twiss, A.C.B. Lovell, givenName surName, Proceedings of the Royal Society of London. Series A. Mathematical and Physical Sciences 242, 300 (1957), https://royalsocietypublishing.org/doi/pdf/10.1098/rspa.1957.0177

21. R.H. Brown, R.Q. Twiss, A.C.B. Lovell, Proceedings of the Royal Society of London. Series A. Mathematical and Physical Sciences 243, 291 (1958), https://royalsocietypublishing.org/doi/pdf/10.1098/rspa.1958.0001

22. T. Martin, R. Landauer, Phys. Rev. B 45, 1742 (1992)

23. M. Büttiker, Phys. Rev. B 46, 12485 (1992)

24. M. Henny, S. Oberholzer, C. Strunk, T. Heinzel, K. Ensslin, M. Holland, C. Schönenberger, Science 284, 296 (1999), https://science.sciencemag.org/content/284/5412/296.full.pdf

25. W.D. Oliver, J. Kim, R.C. Liu, Y. Yamamoto, Science 284, 299 (1999), https://science.sciencemag.org/content/284/5412/299.full.pdf

26. M.P. Anantram, S. Datta, Phys. Rev. B 53, 16390 (1996)

27. T. Martin, Physics Letters A 220, 137 (1996)

28. J. Torrès, T. Martin, G.B. Lesovik, Phys. Rev. B 63, 134517 (2001)

29. G. Lesovik, T. Martin, G. Blatter, The European Physical Journal B - Condensed Matter and Complex Systems 24, 287 (2001)

30. J. Torrès, T. Martin, The European Physical Journal B - Condensed Matter and Complex Systems 12, 319 (1999)

31. G. Deutscher, D. Feinberg, Applied Physics Letters 76, 487 (2000), https://doi.org/10.1063/1.125796

32. P. Recher, E.V. Sukhorukov, D. Loss, Phys. Rev. B 63, 165314 (2001)

33. J. Rech, D. Chevallier, T. Jonckheere, T. Martin, Phys. Rev. B 85, 035419 (2012)

34. A. Haim, E. Berg, F. von Oppen, Y. Oreg, Phys. Rev. Lett. 114, 166406 (2015)

35. A. Haim, E. Berg, F. von Oppen, Y. Oreg, Phys. Rev. B 92, 245112 (2015) 
36. S. Valentini, M. Governale, R. Fazio, F. Taddei, Physica E: Low-dimensional Systems and Nanostructures 75, 15 (2016)

37. E. Bocquillon, V. Freulon, F.D. Parmentier, J.M. Berroir, B. Plaais, C. Wahl, J. Rech, T. Jonckheere, T. Martin, C. Grenier et al., Annalen der Physik 526, 1 (2014), https://onlinelibrary.wiley.com/doi/pdf/10.1002/andp. 201300181

38. J.F. Clauser, Phys. Rev. D 9, 853 (1974)

39. G.B. Lesovik, R. Loosen, Journal of Experimental and Theoretical Physics Letters 65, 295 (1997)

40. U. Gavish, Y. Levinson, Y. Imry, Phys. Rev. B 62, R10637 (2000)

41. R. Aguado, L.P. Kouwenhoven, Phys. Rev. Lett. 84, 1986 (2000)

42. R. Deblock, E. Onac, L. Gurevich, L.P. Kouwenhoven, Science 301, 203 (2003)

43. P.M. Billangeon, F. Pierre, H. Bouchiat, R. Deblock, Phys. Rev. Lett. 96, 136804 (2006)

44. P.M. Billangeon, F. Pierre, H. Bouchiat, R. Deblock, Phys. Rev. Lett. 98, 216802 (2007)

45. J. Basset, H. Bouchiat, R. Deblock, Phys. Rev. Lett. 105, 166801 (2010)

46. E. Zakka-Bajjani, J. Ségala, F. Portier, P. Roche, D.C. Glattli, A. Cavanna, Y. Jin, Phys. Rev. Lett. 99, 236803 (2007)

47. G.B. Lesovik, T. Martin, J. Torrès, Phys. Rev. B 60, 11935 (1999)

48. A. Zazunov, R. Egger, A. Levy Yeyati, Phys. Rev. B 94, 014502 (2016)

49. T. Jonckheere, J. Rech, A. Zazunov, R. Egger, T. Martin, Phys. Rev. B 95, 054514 (2017)

50. Y. Blanter, M. Bttiker, Physics Reports 336, 1 (2000)

51. M. Büttiker, Phys. Rev. Lett. 65, 2901 (1990)

52. D. Bathellier, L. Raymond, T. Jonckheere, J. Rech, A. Zazunov, T. Martin, Phys. Rev. B 99, 104502 (2019)

53. A. Zazunov, M. Creux, E. Paladino, A. Crépieux, T. Martin, Phys. Rev. Lett. 99, 066601 (2007)

54. D. Chevallier, J. Rech, T. Jonckheere, T. Martin, Phys. Rev. B 83, 125421 (2011)

55. T. Martin, in Nanophysics: Coherence and Transport, edited by H. Bouchiat, Y. Gefen, S. Guron, G. Montambaux, J. Dalibard (Elsevier, 2005), Vol. 81 of Les Houches, pp. $283-359$ 